\title{
Neurogenic thoracic outlet syndrome: current diagnostic criteria and advances in MRI diagnostics
}

\author{
Stephen T. Magill, MD, PhD, ${ }^{1}$ Marcel Brus-Ramer, MD, PhD, ${ }^{2}$ Philip R. Weinstein, MD, ${ }^{1}$ \\ Cynthia T. Chin, MD, ${ }^{2}$ and Line Jacques, MD' \\ Departments of ${ }^{1}$ Neurological Surgery and ${ }^{2}$ Radiology and Biomedical Imaging, University of California, San Francisco, \\ California
}

\begin{abstract}
Neurogenic thoracic outlet syndrome (nTOS) is caused by compression of the brachial plexus as it traverses from the thoracic outlet to the axilla. Diagnosing nTOS can be difficult because of overlap with other complex pain and entrapment syndromes. An nTOS diagnosis is made based on patient history, physical exam, electrodiagnostic studies, and, more recently, interpretation of MR neurograms with tractography. Advances in high-resolution MRI and tractography can confirm an nTOS diagnosis and identify the location of nerve compression, allowing tailored surgical decompression. In this report, the authors review the current diagnostic criteria, present an update on advances in MRI, and provide case examples demonstrating how MR neurography (MRN) can aid in diagnosing nTOS. The authors conclude that improved high-resolution MRN and tractography are valuable tools for identifying the source of nerve compression in patients with nTOS and can augment current diagnostic modalities for this syndrome.
\end{abstract}

http://thejns.org/doi/abs/10.3171/2015.6.FOCUS15219

KEY WORDS neurogenic thoracic outlet syndrome; MRI; MR neurogram; DTI; MR tractography; diffusion tensor imaging

$\mathrm{N}$ EUROGENIC thoracic outlet syndrome (nTOS) is a rare condition that consists of a constellation of symptoms resulting from compression of the brachial plexus as it travels from the thoracic outlet to the axilla. Compression can occur between the anterior and middle scalene muscles, underneath a cervical rib, beneath the pectoralis minor tendon, or by fibrous bands anywhere along the course of the plexus. ${ }^{11}$ The most common symptoms, including pain in the neck, shoulder, arm, and hand, are typically unilateral but can also occur bilaterally. ${ }^{13} \mathrm{In}$ one of the largest studies of nTOS, symptoms of lower plexus compression were most common (68\%), followed by symptoms of both upper and lower plexus compression $(20 \%)$ and symptoms of upper plexus compression alone $(12 \%)^{21}$

nTOS must be differentiated from other causes of brachial plexopathy including trauma, mass lesions, vascular lesions, brachial plexitis, and cervical spinal cord or foraminal stenosis. Once these causes have been excluded, it is crucial to differentiate nTOS from "disputed TOS."
Both syndromes have similar symptoms, but diagnosing nTOS requires identification of a direct source of compression, while patients without a clear source of compression fall into the disputed TOS category and may not respond as well to surgery. Given the complexity of diagnosis and the overlap with other pain syndromes, there can often be a delay in nTOS diagnosis and surgical intervention. Current evidence suggests that delaying surgical decompression may lead to irreversible nerve injury and limit potential symptomatic relief and thus emphasizing the need for clear diagnostic criteria to promptly confirm the diagnosis of nTOS rather than waiting for prolonged failure of conservative measures. ${ }^{1}$

Meticulous physical examination of the patient is a basic step in establishing an nTOS diagnosis and requires detailed knowledge of the brachial plexus and upper extremity anatomy and innervation. Common findings can include weakness and/or atrophy in muscle groups corresponding to the compressed trunk or nerve. Maneuvers such as the Wright test (hyperabduction leading to increased pain or

ABBREVIATIONS ACDF = anterior cervical discectomy and fusion; DTI = diffusion tensor imaging; MRC = Medical Research Council; MRN = magnetic resonance neurography; NCS = nerve conduction study; NEE = needle electrode examination; $n T O S$ = neurogenic thoracic outlet syndrome; SPAIR = spectral adiabatic inversion recovery; STIR = short-tau inversion recovery.

SUBMITTED May 1, 2015. ACCEPTED June 10, 2015

INCLUDE WHEN CITING DOI: 10.3171/2015.6.FOCUS15219. 
loss of pulse in vascular TOS) or Adson test (ipsilateral head rotation and inspiration leading to increased pain or loss of pulse in vascular TOS) can support the diagnosis. ${ }^{3}$ Supraclavicular tenderness or a Tinel sign may also be present. The location of the compression can be determined based on physical findings as well. For example, atrophy or weakness of the thenar and hypothenar muscles suggests compression of the lower trunk or medial cord.

Electrophysiological diagnostic confirmation of nTOS is essential to the workup and diagnosis. Electrophysiological studies allow objective differentiation of nTOS from disputed TOS and similar pain syndromes. Tsao et al. conducted electrodiagnostic evaluations in a series of patients with surgically confirmed nTOS, demonstrating that the most important finding was primary axonal loss due to compression. ${ }^{20}$ In that series, the most common source of compression was fibrous bands affecting the lower trunk (C8-T1). Axonal loss was reflected in both a nerve conduction study (NCS) and a needle electrode examination (NEE), with involvement of the median antebrachial cutaneous nerve, primarily supplied by T1 and the most common site of decreased conduction. However, signs of axonal loss can be seen throughout the arm and hand, depending on the location of the compression. Importantly, the NCS and NEE should be performed bilaterally, as most cases are unilateral and the nonaffected side serves as a valuable control, strengthening the diagnosis.

Recently, ultrasound-guided botulinum toxin injection of the anterior scalene muscle has been tested in patients with suspected nTOS. It provides excellent temporary relief in nearly $70 \%$ of patients with nTOS. ${ }^{19}$ A good response to botulinum toxin injection strengthens the diagnosis of nTOS caused by compression from anterior scalene hypertrophy. Given the short duration of action, injected botulinum toxin often does not provide permanent relief, but a positive response to local anesthetic muscle block is associated with relief after surgery. ${ }^{4}$ Patients with compression due to other causes, such as a cervical rib or fibrous band, are unlikely to respond to botulinum toxin injection.

\section{Advances in MR Neurography and Tractography}

High-quality imaging of the brachial plexus is a useful adjunct to confirm the location of compression suggested by the patient history, physical exam, electrodiagnostic studies, and therapeutic trials. Until recently, MRI for TOS evaluation was unable to accurately predict intraoperative findings; new imaging sequences were needed to improve the diagnostic accuracy of MRI. Singh et al. tried to correlate MRI results with intraoperative findings but were successful in identifying surgically proven points of compression by MRI in less than 50\% of patients, demonstrating the need for improved MRI. ${ }^{18}$

MR neurography (MRN) has been available as a specialized imaging study since 1993, when Filler et al. reported methods for producing an "image neurogram" using commercial MRI systems. ${ }^{9}$ This initial neurogram permitted increased conspicuity of neural structures with suppression of signal from surrounding structures by use of the fat suppression technique, heavy T2 weighting, and suppression pulses outside the imaging plane to reduce pulsation artifact from flowing blood. Although the gold standard for diagnosis of peripheral nerve disease is neurological exam and electrodiagnostic study, MRN provides an increasingly important method for evaluating peripheral nerves. MRN allows noninvasive visualization of nerve morphology and signal, permitting precise identification of focal nerve lesions. Modern scanners, particularly those with a magnetic field strength of $3.0 \mathrm{~T}$, provide exquisite anatomical detail and improved visualization of the brachial plexus. Initial studies suggest that these highquality images play an important role in confirming the diagnosis of nTOS. ${ }^{2}$

MRN can be performed using a number of variations on several fundamental techniques. Commonly obtained sequences include standard spin-echo T1- and fast spinecho T2-weighted images with fat suppression. At the University of California, San Francisco, short inversion time or tau inversion recovery (STIR) sequences, which provide intrinsic fat suppression, are also often obtained. A newer alternative to STIR sequences is the T2 spectral adiabatic inversion recovery (SPAIR) preparatory module that can produce increased signal to noise and better selective fat suppression. Finally, a steady-state sequence with $3 \mathrm{D}$ volumetric acquisition is also performed, allowing for greater anatomical detail and the ability to reformat in various planes. Nerves are imaged in 2 planes: longitudinal (parallel) and cross-sectional (perpendicular) to the length of the nerve. These techniques yield images of the nerves known as MR neurograms.

In addition to sequence and scanner selection, the use of transmit-receive coils is important for maximizing the signal-to-noise ratio. This is especially important when imaging peripheral nerves as they course through the limbs. Tightly contoured joint- or limb-specific coils permit a smaller field of view, which produces higherresolution images and higher signal versus background. Minimizing the field of view should be done in consultation with the entire clinical team to focus the exam on the expected region of nerve abnormality. Special imaging techniques, such as phase oversampling, can be used to limit the "wrap-around" artifact that can occur with smaller fields of view. ${ }^{5}$

Recently, the development of tractography using diffusion tensor imaging (DTI) sequences has shown significant potential for noninvasive peripheral nerve imaging. DTI is based on the principles of distinct nonrandom motion of water molecules within highly organized cellular structures such as myelinated nerves and organized nerve bundles. In conjunction with the standard anatomical imaging included in MRN, DTI offers the ability to visualize nerve fascicles, which can have important clinical implications in the diagnosis of peripheral nerve tumor types and in the assessment of peripheral nerve injury and potential regrowth following those injuries ${ }^{8}$

\section{Surgical Management}

The surgical approach chosen for the management of nTOS is determined by the location of the nerve root 
compression. The supraclavicular approach, which is most commonly used to treat patients with nTOS, involves scalenectomy and, if indicated, cervical rib resection..$^{15} \mathrm{In}$ this approach, an incision is made above the clavicle posterior to the sternocleidomastoid and anterior to the trapezius muscles. Dissection proceeds until the phrenic nerve is identified, traveling over the anterior scalene muscle, followed by identification of C-5 and then C- 6 . The upper, middle, and lower trunks of the brachial plexus are identified, and then the compressing muscle or rib can be resected to decompress the neural elements. When performed in patients with clearly localized compression, this approach can provide significant improvement in pain and disability. 6,23

If the compression is caused by a distal structure, an infraclavicular or transaxillary approach can be used. ${ }^{11}$ The transaxillary approach is most commonly used to treat vascular TOS and less often for nTOS. ${ }^{7}$ The infraclavicular approach is well suited for compression caused by the pectoralis minor muscle. ${ }^{16,22}$ In the infraclavicular approach, an incision is made in the deltopectoral groove. The pectoralis major muscle is retracted, and the insertion site of the pectoralis minor muscle on the coracoid process can be visualized. Once the neurovascular bundle is dissected free from any adhesions, the pectoralis minor tendon can be coagulated and cut, providing good decompression.

\section{Illustrative Cases}

Three case examples are presented, demonstrating the use of high-resolution neurograms for confirming the diagnosis of nTOS and their role in localizing the site of neural compression.

\section{Case 1}

A 56-year-old woman presented with 10-15 years of right hand and arm weakness as well as tingling in the fourth and fifth digits. She had a carpal tunnel release procedure 10 years prior and a C3-6 anterior cervical discectomy and fusion (ACDF) 3 years before presenting to our clinic. However, her hand weakness persisted despite the decompression. On physical exam, she had weakness (Medical Research Council [MRC] Grade 4-/5) in her right flexor carpi ulnaris, right abductor pollicis brevis, right opponens pollicis, and right interossei muscles. The Tinel sign was positive over the right wrist. She had decreased sensation to pin prick and temperature over the right medial palm, the fourth and fifth digits, and the medial forearm. She had a positive Adson test and muscle atrophy of the thenar and hypothenar muscles. Decreased amplitudes on electrodiagnostic studies were consistent with axonal loss in the lower trunk or the C-8 nerve root. MRN demonstrated focally increased signal within the right brachial plexus, affecting the nerve roots just proximal to the interscalene space (Fig. 1A and B). Preoperative MR tractography visualized the impression caused by the scalene muscle on the right C-6 and the C-8 nerve roots (Fig. 1C). The patient underwent anterior scalenectomy via a supraclavicular approach (Fig. 1D). At the 1-month follow-up, the patient reported improved function in her
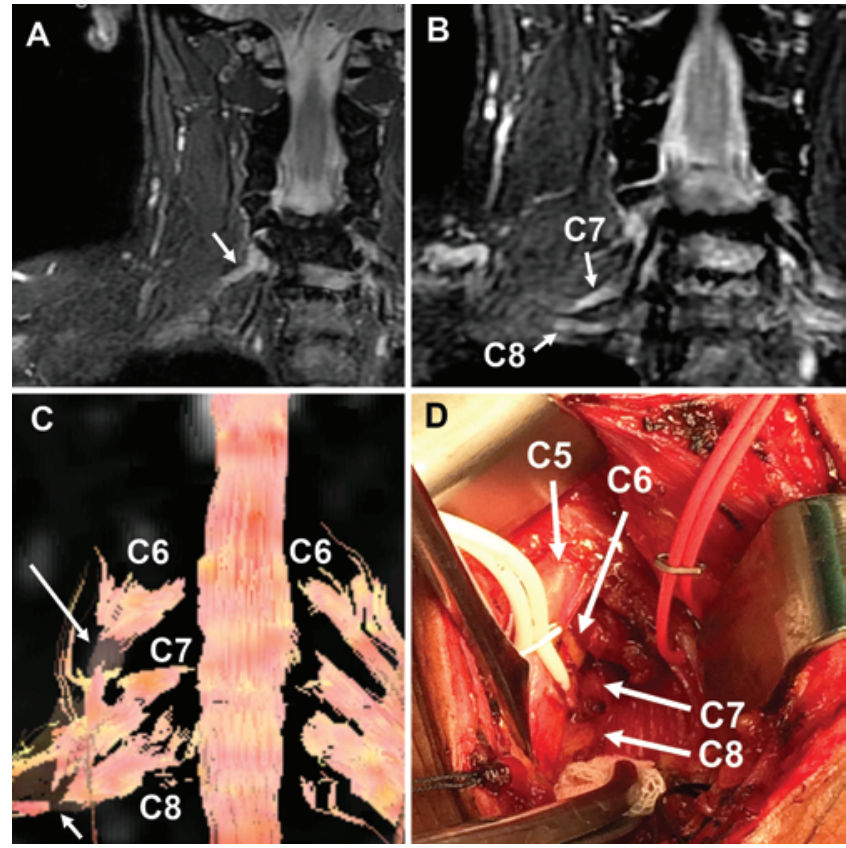

FIG. 1. Case 1. Representative images showing compression between the anterior and middle scalene muscles. A: Coronal STIR-weighted MR neurogram shows increased signal and expansion in the C-6 nerve root as it enters the interscalene space (arrow). B: Coronal STIR-weighted MR neurogram shows increased signal in C-7 and C-8 nerve roots as they enter the interscalene space. C: MR tractography shows impression (arrows) of the scalene muscle (red band on tractography) on C- 6 as it enters the interscalene space and on C- 8 where it is compressed by scalene muscle. The tract size is decreased distal to the site of compression. D: Intraoperative photo demonstrates the decompressed C5-8 nerve roots, the upper trunk (white vessel loop), and the phrenic nerve (red vessel loop) after anterior scalenectomy and neurolysis.

right hand; upon examination, her intrinsic right hand strength had improved to MRC Grade 4/5, and she had full range of motion in her shoulder.

\section{Case 2}

A 58-year-old woman presented with intractable neck and left-arm pain that had persisted for more than 20 years. She had previously undergone C5-7 ACDF, followed by removal of C5-7 hardware and C3-5 ACDF. However, she had a persistent left $\mathrm{C}-8$ radiculopathy that was exacerbated by shoulder and arm movement. She had no muscle atrophy or weakness. Noncontrast cervical spine CT showed a left C-7 cervical rib formation (Fig. 2A). MRN showed compression of the C-8 nerve root and kinking of the nerve root as it passed inferiorly to the cervical rib (Fig. 2B and C). Electrodiagnostic studies demonstrated C-8 axonal loss. The patient underwent resection of the C-7 cervical rib with decompression of the C-8 nerve root. At the 4-month follow-up, her left C-8 radiculopathy had resolved. She was pain free and had full strength and range of motion on motor testing in her left deltoid, biceps, triceps, wrist extensors, and dorsal interosseous muscles.

\section{Case 3}

A 53-year-old man presented with pain and numbness 

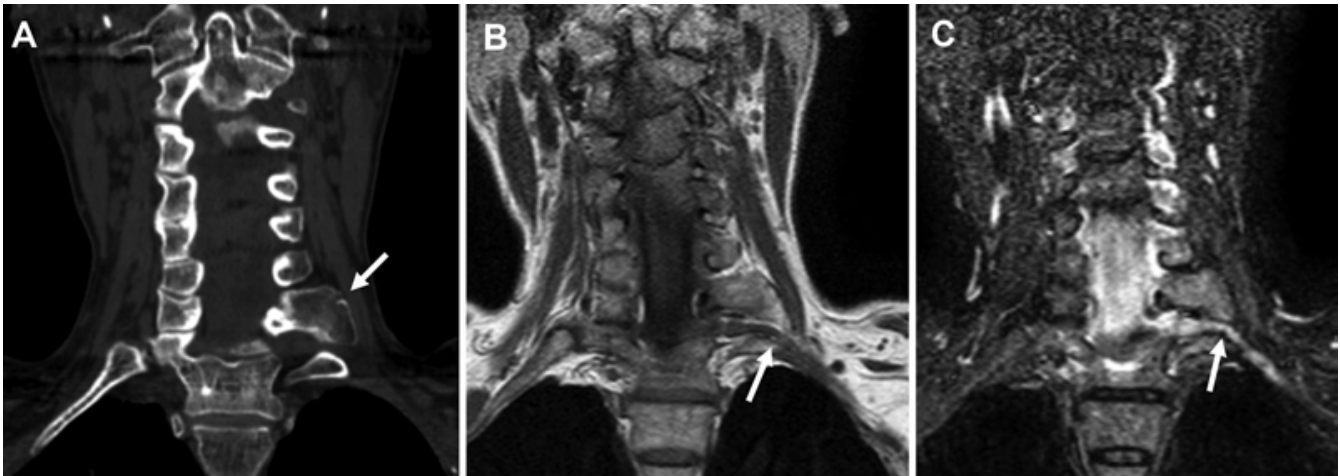

FIG. 2. Case 2. Representative images showing cervical rib-induced C-8 compression. A: Coronal CT scan shows the left C-7 cervical rib (arrow). B: Coronal T1-weighted MR neurogram shows kinking of the C-8 nerve root (arrow) underneath the cervical rib. C: Coronal STIR sequence shows kinking of the C-8 nerve root (arrow) underneath the C-7 cervical rib.

in the right fourth and fifth digits as well as atrophy of the thenar and hypothenar muscles. On exam, he had muscle weakness (MRC Grade 4-/5) in his right grip, finger extension, and interosseous muscles. Electrodiagnostic studies demonstrated $\mathrm{C}-8$ axonal loss that localized to the nerve root level. MRN showed a kink in the nerve (Fig. $3 \mathrm{~A}$ and $\mathrm{B})$. T1-weighted images revealed a fibrous band attached to the right $\mathrm{C}-7$ transverse process and passing over the nerve root causing compression (Fig. 3C). The patient underwent resection of the fibrous band and anterior and middle scalenectomy with neurolysis. Postoperative MRN demonstrated release of the fibrous band and straightening
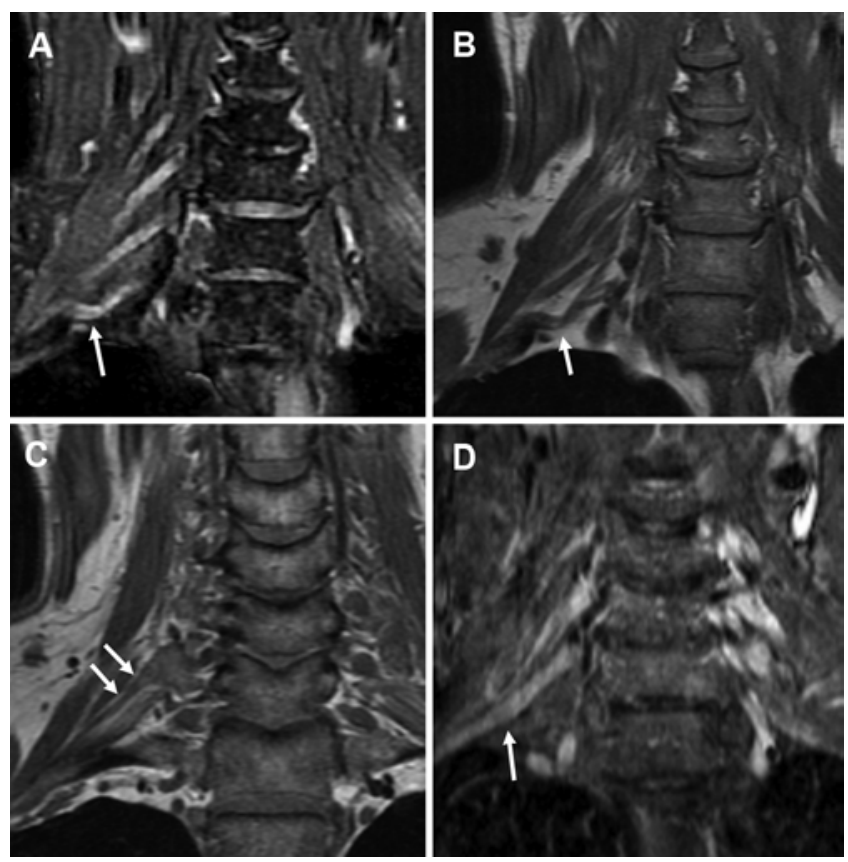

FIG. 3. Case 3. Representative images showing a fibrous band causing kinking of the C-8 nerve root. A: Coronal STIR MR neurogram shows a kink in the C-8 nerve root (arrow). B: Coronal T1-weighted MR neurogram shows the same kink in the C-8 nerve root (arrow). C: Coronal T1-weighted MR neurogram shows a small thin band (arrows) extending from the asymmetrically enlarged C-7 transverse process that was overlying the C-8 nerve root. D: Postoperative coronal STIR-weighted MR neurogram shows straightening of C-8 nerve root (arrow) after the fibrous band has been resected. of the C-8 nerve (Fig. 3D). At the 1-month follow-up, the patient reported subjective improvement in sensation in the C-8 dermatome, resolution of his pain, improved hand function, and subjective improvement in atrophy, and he displayed MRC Grade 4/5 strength on physical examination in his grip, finger extension, and interosseous muscles.

\section{Discussion}

nTOS can be difficult to diagnose and is a source of significant pain and disability for patients. Accurate diagnosis and differentiation from other sources of neck pain and upper extremity weakness, most often due to cervical disc disease and spondylosis, is essential for determining the most appropriate treatment for each patient. The diagnosis is supported by a thorough patient history, meticulous physical exam, electrodiagnostic studies, and imaging studies (Table 1). Surgical treatment for nTOS can result in long-term improvement in quality of life. ${ }^{14}$ However, an inaccurate diagnosis can lead to unnecessary surgery without benefit.

The cases presented here demonstrate the utility of MRN to augment patient history, physical exam, and electrodiagnostic studies. The usefulness of this imaging technique has been demonstrated by other groups as well, including authors of a recent case report of a fibrous band-induced nTOS that was identified by MRI, allowing focused resection with a good outcome.24

In addition to contributing to the initial diagnosis, im-

TABLE 1. nTOS diagnostic criteria

\begin{tabular}{lc}
\hline Symptoms & $\begin{array}{c}\text { Disabling upper extremity, shoulder, or neck pain } \\
\text { (typically unilat); may or may not improve w/ } \\
\text { botulinum toxin injection }\end{array}$ \\
\hline Physical exam & $\begin{array}{c}\text { Weakness in neurologically discreet distributions; } \\
\text { muscle atrophy corresponding to compressed } \\
\text { trunk }\end{array}$ \\
\hline $\begin{array}{c}\text { Electrodiagnostic } \\
\text { studies }\end{array}$ & $\begin{array}{c}\text { Axonal loss on NCS/NEE } \\
\text { Imaging studies }\end{array}$ \\
& $\begin{array}{c}\text { MR neurography showing site of compression; MR } \\
\text { tractography showing axonal loss distal to com- } \\
\text { pression; MRI showing cervical spine negative } \\
\text { for cord or foraminal stenosis }\end{array}$ \\
\hline
\end{tabular}


proved MRN and tractography may be useful in cases of recurrent and/or persistent nTOS. Prior studies have shown that CT can be useful for identifying structural causes of persistent TOS symptoms, but it still fails to correlate with operative findings in $15 \%$ of patients. The superior anatomical detail seen on MR neurograms helps physicians in the workup and evaluation of these patients..$^{10}$

While our study has focused primarily on the role of MRI, ultrasonography may also serve as an excellent diagnostic tool for nTOS when performed by a skilled practitioner, particularly for evaluating neural compression during dynamic movements, such as with the Adson or Wright test. ${ }^{12,17}$ Ultrasound may be an especially useful adjunct for patients with disputed TOS where compression is equivocal on MR neurograms or electrodiagnostic studies.

\section{Conclusions}

In conclusion, we have presented a concise review of nTOS diagnosis and imaging, focusing on the diagnostic criteria and utility of MRI. Recent advances in MRN are providing detailed images that show localization and visualization of nerve root compression, and thereby augmenting data obtained from a patient history, a physical exam, and electrodiagnostic studies. MRN improves diagnostic certainty, allows a tailored surgical approach for each lesion, and facilitates safe and successful surgical decompression, with the ultimate goal of improving each patient's symptoms and quality of life.

\section{References}

1. Al-Hashel JY, El Shorbgy AAMA, Ahmed SF, Elshereef RR: Early versus late surgical treatment for neurogenic thoracic outlet syndrome. ISRN Neurol 2013:673020, 2013

2. Baumer P, Kele H, Kretschmer T, Koenig R, Pedro M, Bendszus M, et al: Thoracic outlet syndrome in 3T MR neurography-fibrous bands causing discernible lesions of the lower brachial plexus. Eur Radiol 24:756-761, 2014

3. Braun RM: Thoracic outlet syndrome: a primer on objective methods of diagnosis. J Hand Surg Am 35:1539-1541, quiz 1541,2010

4. Caputo FJ, Wittenberg AM, Vemuri C, Driskill MR, Earley JA, Rastogi R, et al: Supraclavicular decompression for neurogenic thoracic outlet syndrome in adolescent and adult populations. J Vasc Surg 57:149-157, 2013

5. Chhabra A, Flammang A, Padua A Jr, Carrino JA, Andreisek G: Magnetic resonance neurography: technical considerations. Neuroimaging Clin N Am 24:67-78, 2014

6. Dalbayrak S, Yaman O, Yilmaz M, Yilmaz T: Supraclavicular surgical approach for thoracic outlet syndrome: 10 years of experience. Turk Neurosurg 24:867-872, 2014

7. Degeorges R, Reynaud C, Becquemin JP: Thoracic outlet syndrome surgery: long-term functional results. Ann Vasc Surg 18:558-565, 2004

8. Eppenberger P, Andreisek G, Chhabra A: Magnetic resonance neurography: diffusion tensor imaging and future directions. Neuroimaging Clin N Am 24:245-256, 2014

9. Filler AG, Howe FA, Hayes CE, Kliot M, Winn HR, Bell BA, et al: Magnetic resonance neurography. Lancet 341:659-661, 1993

10. Greenberg JI, Alix K, Nehler MR, Johnston RJ, Brantigan $\mathrm{CO}$ : Computed tomography-guided reoperation for neurogenic thoracic outlet syndrome. J Vasc Surg 61:469-474, 2015
11. Klaassen Z, Sorenson E, Tubbs RS, Arya R, Meloy P, Shah R, et al: Thoracic outlet syndrome: a neurological and vascular disorder. Clin Anat 27:724-732, 2014

12. Lapegue F, Faruch-Bilfeld M, Demondion X, Apredoaei C, Bayol MA, Artico H, et al: Ultrasonography of the brachial plexus, normal appearance and practical applications. Diagn Interv Imaging 95:259-275, 2014

13. Povlsen B, Hansson T, Povlsen SD: Treatment for thoracic outlet syndrome. Cochrane Database Syst Rev 11:CD007218, 2014

14. Rochlin DH, Gilson MM, Likes KC, Graf E, Ford N, Christo PJ, et al: Quality-of-life scores in neurogenic thoracic outlet syndrome patients undergoing first rib resection and scalenectomy. J Vasc Surg 57:436-443, 2013

15. Sanders RJ, Annest SJ: Technique of supraclavicular decompression for neurogenic thoracic outlet syndrome. J Vasc Surg 61:821-825, 2015

16. Sanders RJ, Annest SJ, Goldson E: Neurogenic thoracic outlet and pectoralis minor syndromes in children. Vasc Endovascular Surg 47:335-341, 2013

17. Simon NG, Ralph JW, Chin C, Kliot M: Sonographic diagnosis of true neurogenic thoracic outlet syndrome. Neurology 81:1965, 2013

18. Singh VK, Jeyaseelan L, Kyriacou S, Ghosh S, Sinisi M, Fox M: Diagnostic value of magnetic resonance imaging in thoracic outlet syndrome. J Orthop Surg (Hong Kong) 22:228-231, 2014

19. Torriani M, Gupta R, Donahue DM: Botulinum toxin injection in neurogenic thoracic outlet syndrome: results and experience using a ultrasound-guided approach. Skeletal Radiol 39:973-980, 2010

20. Tsao BE, Ferrante MA, Wilbourn AJ, Shields RW: Electrodiagnostic features of true neurogenic thoracic outlet syndrome. Muscle Nerve 49:724-727, 2014

21. Urschel HC Jr, Razzuk MA: Neurovascular compression in the thoracic outlet: changing management over 50 years. Ann Surg 228:609-617, 1998

22. Vemuri C, Wittenberg AM, Caputo FJ, Earley JA, Driskill MR, Rastogi R, et al: Early effectiveness of isolated pectoralis minor tenotomy in selected patients with neurogenic thoracic outlet syndrome. J Vasc Surg 57:1345-1352, 2013

23. Yaseen Z, Baram A: Neurogenic thoracic outlet syndrome treatment by the supraclavicular approach. Asian Cardiovasc Thorac Ann 22:193-196, 2014

24. Yildizgören MT, Ekiz T, Kara M, Yörübulut M, Ozçakar L: Magnetic resonance imaging of a fibrous band causing true neurogenic thoracic outlet syndrome. Am J Phys Med Rehabil 93:732-733, 2014

\section{Disclosure}

The authors report no conflict of interest concerning the materials or methods used in this study or the findings specified in this paper.

\section{Author Contributions}

Conception and design: Jacques, Magill, Weinstein, Chin. Acquisition of data: Jacques, Magill, Brus-Ramer, Chin. Analysis and interpretation of data: all authors. Drafting the article: Magill, Brus-Ramer. Critically revising the article: all authors. Reviewed submitted version of manuscript: all authors. Approved the final version of the manuscript on behalf of all authors: Jacques.

\section{Correspondence}

Line Jacques, Department of Neurological Surgery, University of California, San Francisco, 505 Parnassus Ave., M779, San Francisco, CA 94131.email: line.jacques@ucsf.edu. 